Hence, quadratic forms exist for which the corresponding values of $\rho$ satisfy these inequalities. However, no such form has ever been constructed for large values of $n$.

The problem of the closest regular (latticed) packing of spheres in $R_{n}$ is equivalent to the problem of finding that positive definite quadratic form in $n$ variables and of given determinant, say $D=1$, whose least value, other than zero, is the highest possible for the given $n$ and $D\left(\mathrm{M}_{4}\right.$, page $74 \mathrm{ff}$.). The ratio of the space occupied by spheres packed in regular layers in a large cube, say, to the volume of the whole cube, is indeed the number $\rho$ defined above.

The author has recently proved, by an essentially different method, that no matter how the spheres be packed in a large volume $V$, in a "regular" fashion or not, the ratio of the space occupied by the spheres to the whole volume $V$ is

$$
<\frac{n / 2+1}{2^{n / 2}} \text {. }
$$

It may be of interest to note in passing that it follows from the inequalities (1) satisfied by $\rho$ for certain (though unknown) quadratic forms, that the shot-pile packing of spheres, though the closest packing in space of two dimensions and presumably also in space of three dimensions, is very far from being the closest packing in space of a large number of dimensions $\left(\mathrm{M}_{4}\right.$, page 95$)$.

\title{
APPLICATIONS OF THE GEOMETRY OF NUMBERS TO ALGEBRAIC NUMBERS.
}

BY PROFESSOR L. E. DICKSON.

(Read at the Chicago Symposium of the American Mathematical Society, March 28, 1919.)

1. The geometry of numbers not only furnishes a concrete geometric image of certain fundamental theorems on algebraic numbers, but also provides a new and attractive method of proving important theorems on algebraic fields. For the sake of concreteness we shall restrict attention to the typical case of the cubic field $F(\theta)$, which is composed of the numbers 
$r=X+Y \theta+Z \theta^{2}$, where $X, Y, Z$ are rational numbers, and $\theta$ is a root of a cubic equation with integral coefficients, that of $\theta^{3}$ being unity. A root of any such equation is called an integral algebraic number.

2. Basis of a Field.- While the above number $r$ is an integral algebraic number if $X, Y, Z$ are integers, the converse need not be true. However, there exist integral algebraic numbers $\omega_{1}, \omega_{2}, \omega_{3}$ of the field $F(\theta)$ such that the linear functions of $\omega_{1}, \omega_{2}, \omega_{3}$ with integral coefficients give all the integral algebraic numbers of the field. The essential idea of the geometric proof consists in showing that the points $(X, Y, Z)$ which correspond to the integral algebraic numbers $r$ of our field form a lattice determined by the fundamental parallelopiped whose edges $O A, O B, O C$ are defined as follows: Of the points on the positive $X$-axis which correspond to integral algebraic numbers of $F(\theta)$, let $A$ be the one nearest the origin $O$ and distinct from $O$. Of the points in the $X Y$-plane having $Y>0$ and corresponding to integral algebraic numbers of $F(\theta)$, let $B$ be a point lying as near the $X$-axis as possible. Finally, of the points with $Z>0$ and corresponding to integral algebraic numbers of our field, let $C$ be one as near as possible to the $X Y$-plane.

3. Basis of an Ideal.-A set of integral algebraic numbers of a field is called an ideal if the sum and difference of any two equal or distinct numbers of the set are numbers of the set and if the product of any number of the set by any integral algebraic number of the field equals a number of the set. Consider the lattice whose points correspond to the integral algebraic numbers $x \omega_{1}+y \omega_{2}+z \omega_{3}$ of our field, $x, y, z$ being integers. The points of this lattice which correspond to the numbers of any ideal form a new lattice with a fundamental parallelopiped determined by $O A_{1}, O A_{2}, O A_{3}$. If $\alpha_{i}$ is the number corresponding to the point $A_{i}$, the numbers of the ideal are the linear combinations, with integral coefficients, of $\alpha_{1}, \alpha_{2}, \alpha_{3}$, which therefore form a basis of the ideal.

4. Units of a Field.-By a unit $\epsilon$ is meant an integral algebraic number which divides unity, so that $1 / \epsilon$ equals an integral algebraic number. Dirichlet* stated, but did not publish a detailed proof of, the following fundamental theorem: If among the fields conjugate to a field $F$ of degree $m, r$ are real and the remaining $m-2 r=2 c$ form $c$ pairs of conjugate

* Sitzungsber. Akad. Wiss. Berlin, 1846, pp. 103-7; Werke, I, p. 639 (cf. p. 622, p. 630). 
imaginary fields, there exist $s=r+c-1$ units $\epsilon_{1}, \ldots, \epsilon_{s}$ in $F$ such that every unit $\epsilon$ of $F$ can be expressed in one and but one way in the form

$$
\epsilon=\rho \epsilon_{1}^{a_{1}} \cdots \epsilon_{s}^{a_{s}}
$$

where $a_{1}, \ldots, a_{s}$ are rational integers, positive, negative or zero, while $\rho$ is a root of unity belonging to the field $F$. Kronecker* gave a proof along the lines suggested by Dirichlet. Another algebraic proof had been given by Dedekind. $\dagger$

Minkowskił gave a new proof which is largely algebraic, but makes some use of his geometric results. Later, he gave§ a more purely geometric form to his proof, confining his discussion to the typical cases of cubic and quartic fields. This proof makes use of various ideas and results of the geometry of numbers and shows the power and attractiveness of the latter subject.

5. Classes of Ideals.-In his proof of the finiteness of the number of classes of ideals in a cubic field, Minkowskil| made use of his results on the upper bound of a product of three linear forms. The process enables us actually to find representatives of the various existing classes of ideals. This problem would be simplified by the discovery of more exact upper bounds.

\section{PRODUCTS OF SKEW-SYMMETRIC MATRICES.}

BY PROFESSOR A. A. BENNETT.

In the March (1919) number of the Bulletin occurs (page 281) the following: The philosophical faculty of the University of Berlin announces the following prize problem: "To determine by means of the theory of elementary divisors, the criteria that a given matrix be capable of representation as the composition of two skew-symmetric matrices." A dis-

* Comptes Rendus, Paris, vol. 96, 1883; vol. 99, 1884; Werke, III pp. $1-30$.

$\dagger$ Dirichlet-Dedekind, Zahlentheorie, ed. 2, 1871, § 166, pp. 471-9; ed. $3,1879, \S 177$, pp. 555-567; ed. 4, 1894, § 183, pp. 590-603. Cf. Hilbert, Jahresbericht der Deutschen Math.-Vereinigung, vol. 4, 1894-5, pp. 214-222.

$\ddagger$ Geometrie der Zahlen, 1896, pp. 135-147.

Diophantische Approximationen, 1907, pp. 133-148.

II Diophantische Approximationen, pp. 162-167. 